\title{
Thermal Diffusion Performance of a Diffuser by various Guide Vanes configurations
}

\author{
Aja O. Chikere ${ }^{1}$, Hussain H. Al-Kayiem ${ }^{2, ~ *}$, and Zainal Ambri A. Karim² \\ ${ }^{1}$ Mechanical Engineering Department, Curtin University, Sarawak Malaysia. \\ ${ }^{2}$ Mechanical Engineering Department, Universiti Teknologi PETRONAS, 32610 Bandar Seri \\ Iskandar, Perak, Malaysia.
}

\begin{abstract}
The use of vane-less diffuser with large diffusion angle has shown a setback in the diffusion process of high temperature working fluids. The hot gas flow was characterized as a jet-like flow. This paper presents problem, encountered practically, using a vane-less diffuser with large diffusion angle and how the problem is solved by CFD simulation. The investigated thermal diffuser has a length of $0.3 \mathrm{~m}$, an inlet to outlet crosssectional area ratio of 1:25 and diffusion angle of $115.44 \mathrm{o}$. To resolve the jet-like flow problem and poor distribution of the flow temperature at the diffuser outlet, the study suggested the use of guide-vanes into the diffuser. The study employed CFD simulation by ANSYS-FLUENT software to analyze the flow and thermal process in the diffuser. Three different shapes of guide vanes; block-shaped, oval-shaped and airfoil-shaped were considered in this study and at different vanes diffusion angles, as well as vane-less case, which was adopted as the bench mark case. The simulation results of the velocity, temperature and pressure at the diffuser outlet were compared for all cases. It was found that the guide vanes with symmetrical airfoil profile provided the best performance with most uniform distribution at the outlet of the diffuser. Also, the airfoil-shaped guide vanes resulted in lower pressure losses compared to the block-shaped and oval-shaped guide vanes. According to the analysis results, the diffuser was redesigned to improve the diffusion and temperature distribution across the diffuser outlet.
\end{abstract}

\section{Introduction}

Diffusers are common sudden or gradual expansion component employed in piping/fluid flow processes to decelerate flow and recover static pressure, thus converts kinetic energy into pressure energy. The angle of diffusion of a diffuser determines the flow regime characteristics of the diffused fluid with respect to the diffuser non-dimensional length (inletoutlet length (N)/diffuser inlet diameter (d)) Reneau et al. [1]. A variation in diffuser area ratio and the diffusion angle with respect to the diffuser non-dimensional length determines the flow regime developed within the diffuser. In the design of fluid flow system such as diffusers, computational fluid dynamics (CFD) techniques have been employed by researchers, scientists and engineers as a tool to estimate a real practical process, thus

\footnotetext{
*Corresponding author: hussain_kayiem@utp.edu.my
} 
reducing the total dependence on experimental measurements. Similarly, the use of guide vanes in flow process has found applications in air-conditioning systems, micro turbines, and some turbo-machines.

Studies were conducted by Jiao et al. [2] in the design and performance of various kinds of diffusers for enhances performance include a 3-D numerical study on the effect of diffuser vanes on the performance of a turbocharger compressor system for light-duty diesels, encompassing the airflow geometry from impeller inlet to volute outlet. The results of the study inferred that the angle of the diffuser vane has significant influence on the compressor operating range when compared with vane-less designs. Marconcini et al. [3] investigated the flow field of a high pressure ratio centrifugal compressor for turbocharger application with a compressor consists of a double-splitter impeller followed by a vane diffuser and found that there was improved distribution with the guide vanes. Gopaliya et al. [4] investigated the effect of inlet and outlet of an S-shaped diffuser in two different planes of $90^{\circ} / 90^{\circ}$ turn. The obtained results from this research show reduced outlet pressure recovery accompanied with increase in non-uniformity at the diffuser outlet due to offset. Anand and Sandeep [5] studied the fluid flow characteristics in Y-shaped diffusing ducts of two inlets and one outlet using three Y-shaped diffusing ducts of same curvilinear length and area ratio but with varying angle of turn at $15 \mathrm{o}, 22.5^{\circ}$ and $30^{\circ}$. Their results showed that the velocity pattern at the exit is more uniform for the diffusing duct with smaller angle of turn which is $15^{\circ} / 15^{\circ}$ as a result of reduced wake at the meeting point of the two inlet ducts. Similarly, the coefficient of static pressure recovery decreased with increase in angle of turn which led to increase in total pressure loss with increase in the angle of turn. Similar observation was recorded in the study of Y-shaped diffuser of Gopaliya and Chaudhary [6]. Arndt et al [7] conducted experimental investigation of rotor-stator interaction in a centrifugal pump with several vanned diffusers. They observed that there were large pressure fluctuations on the diffuser vanes and the impeller blades as the total pressure rise across the pump. The results showed that the largest pressure fluctuations on the diffuser vanes occurred at the suction side of the vane near the vane leading edge, whereas on the impeller blades the largest fluctuations occurred at the blade trailing edge. Aziz et al. [8] investigates experimentally and numerically airflow characteristics of vortex, round, and square ceiling diffuser and its effect on the thermal comfort in a ventilated room. The results showed different air flow pattern and diffusion rate.

The above mentioned investigations were conducted for diffusers with small diffusion angles between $0^{\circ}$ to $30^{\circ}$. Previous studies did not consider cases where the diffusion angle from the centreline above $30^{\circ}$. This work focuses on solving an experimental problem encountered using a diffuser with large diffusion angle for discharge of high temperature, high velocity flue gas to a flow channel inclined to the outlet of the diffuser at $45^{\circ}$.

\section{Problem identification}

An experimental set for hybrid solar/flue gas chimney model was designed and fabricated. The flue gas was supplied from experimental gas turbine demonstrate. Schematic of the setup is shown in Figure 1. 


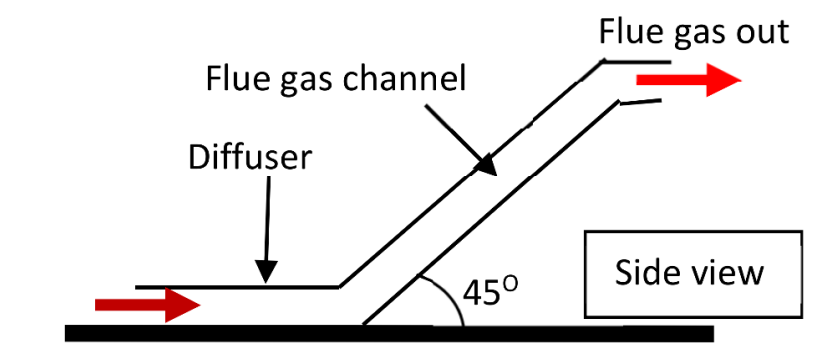

Flue gas in

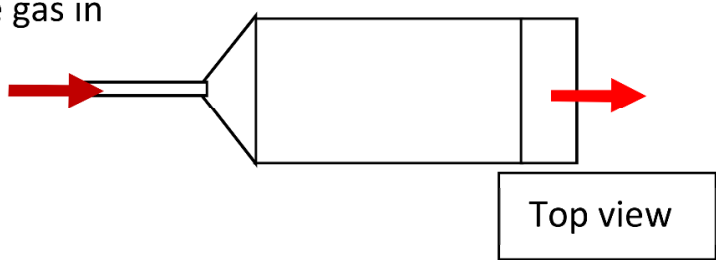

Fig. 1. Schematic diagram of the flue gas diffuser, flue gas channel and the flue gas outlet.

The diffuser has an inlet to outlet length of $0.3 \mathrm{~m}$, inlet pipe diameter of $0.05 \mathrm{~m}$, outlet diffusion width of $1 \mathrm{~m}$, inlet cross-sectional area of $0.002 \mathrm{~m}^{2}$, outlet cross-sectional area of $0.05 \mathrm{~m}^{2}$. The flow process was characterized of poor diffusion and jet-like flow which impacted on the wall of the inclined flow channel at the diffuser outlet which caused damage to the flow channel as shown in Figure 2. This study was conducted to solve the problems of jet-like flow, poor diffusion and uneven thermal distribution across the flow channel using CFD technique by ANSYS-FLUENT software.

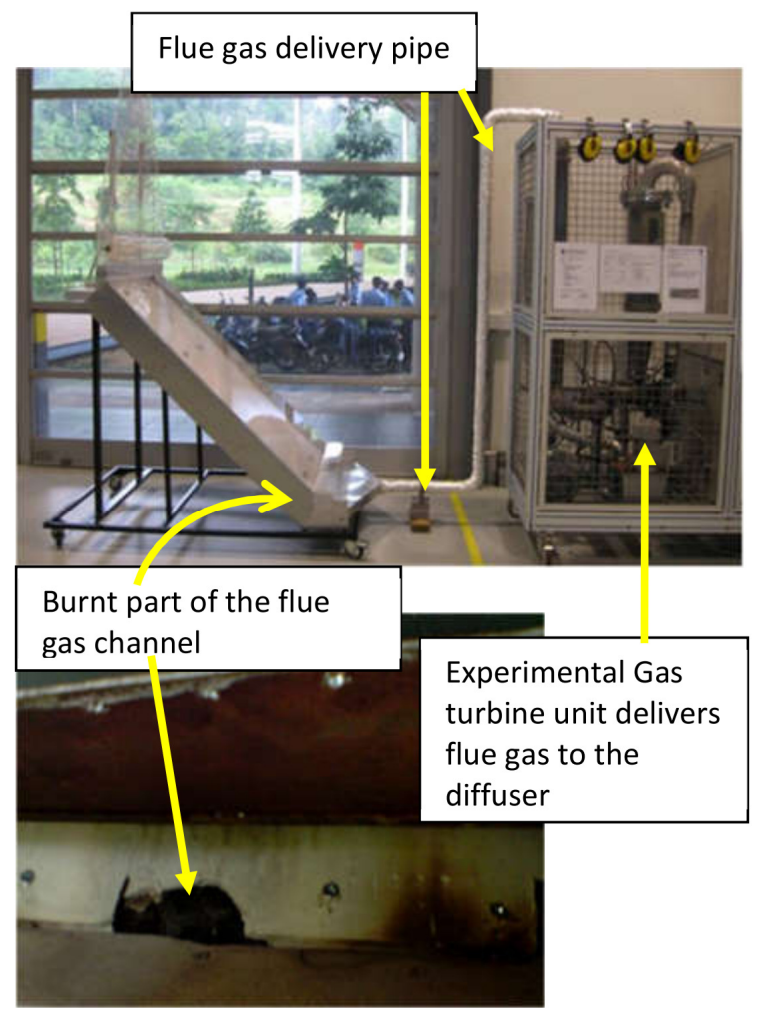

Fig. 2. Burnt part of the flue gas channel due to jet-like flow in the diffuser. 


\subsection{Model description and governing equations}

The diffuser configuration in the present study is shown schematically in Figure 3. The 2-D configuration has inlet diameter of $0.05 \mathrm{~m}$, diffusion angle of $115.44^{\circ}\left(57.72^{\circ}\right.$ from centreline of the diffuser inlet), $0.3 \mathrm{~m}$ axial-length (x-direction) between the diffuser inlet and outlet, and outlet length (y-direction) $1.0 \mathrm{~m}$.

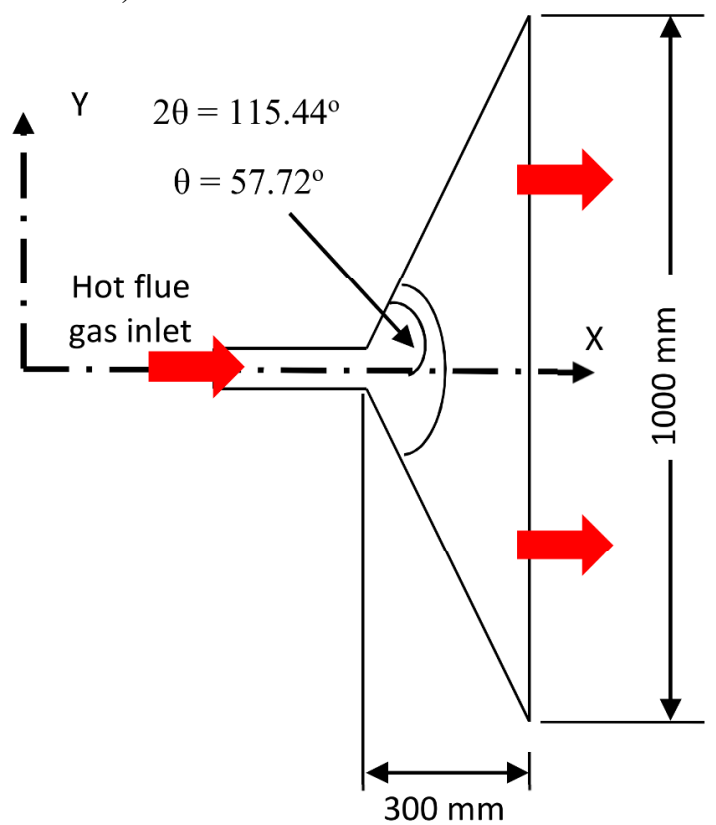

Fig. 3. Schematic diagram of the 2-D model of the diffuser.

The system has inlet to outlet aspect ratio of 1:20 and inlet to outlet area ratio of 1:25. The design was employed to diffuse high temperature flue-gas into a flue gas flow channel with desire of uniform heat transfer to the top wall of the channel which assumes the function of heat exchanger/absorber plate as part of waste heat recovery system. It was found during the experimental process that the diffuser created a jet-like discharge instead of uniform diffusion at the diffuser outlet which was the intent of the design. To solve this problem, the system was studied numerically using computational fluid dynamics (CFD) software (ANSYS-FLUENT) to investigate the flow process and modify the model for improved performance. The diffuser 2-D geometry was developed and the fluid flow and thermal field were simulated using ANSYS-FLUENT commercial software for vane-less and guide-vaned diffuser models. The guide vane shapes employed in this study are block-shaped, oval-shaped and airfoil-shaped guide-vanes which were studied at different angular arrangements as distributors.

\subsection{Basic equations of the diffuser model thermal field and fluid flow}

The 2-D steady state Navier-Strokes-equations (continuity, momentum and energy equations) which describe the flow in the diffuser have been solved iteratively by the software to predict the velocity, temperature and the pressure in the flow field of the diffuser. The simulation employed the Reynolds-Averaged Navier-Stokes (RANS) model which solves the equations for two-dimensional. Based on the velocity of the flue gas, Reynolds value demonstrated that the flow was turbulent. Hence turbulence was considered in the simulation using standard $k-\varepsilon$-turbulence model with consideration of wall treatment functions. It has 
been reported that the near wall treatment of the turbulence model has great influence on the computation of turbulence in flow systems (Esch and Menter [9], Menter et al. [10], and Drabek [11]). In this study, the flue gas temperature was $473 \mathrm{~K}$ and the flow is turbulent; therefore the turbulence and energy dissipation, respectively. Since the equations are well known, and are widely presented in the literature and the software guide, they are not presented here, and the reader may get them from many sources, including the values of the constants in the transport $k-\varepsilon$ models.

\subsection{Diffuser performance parameters}

The main objective of a diffuser in most engineering systems is to increase the static pressure at the diffuser outlet. In this model, the uniform distribution of the flue gas across the diffuser outlet width is a very important factor. To describe the performance of a diffuser, pressure recovery factor (coefficient of static pressure gain) is used. Pressure recovery factor is the increase in fluid static pressure that occurs as fluid moves from small cross-sectional area to a larger cross-sectional area (outlet). The pressure recovery factor for incompressible flow can be written as Equation 1. This parameter represents the kinetic energy that has been converted into pressure energy due to diffusing action.

$$
C_{p s}=\frac{2\left(p_{f g-\text { outlet }}-p_{f g-\text { inlet }}\right)}{\rho_{f g} u_{f g-\text { inlet }}^{2}}
$$

Where $p_{f g \text {-inlet }}$ and $p_{f g \text {-outlet }}$ indicate the averaged pressure at inlet and outlet respectively, $u_{f g \text {-inlet }}$ represent the mass averaged velocity of flue gas into the diffuser, and $\rho_{f g}$ stands for the density of the flue gas.

For an ideal condition, pressure recovery factor of a diffuser depends mainly on the area ratio. It is a situation where the kinetic energy of the flow is converted to pressure without losses, thus Equation 1 becomes

$$
C_{p s-\text { ideal }}=1-\frac{1}{A R^{2}}
$$

When the ideal pressure recovery of a diffuser is determined, the diffuser effectiveness can be evaluated. The effectiveness of a diffuser, $\zeta_{d}$, represents the pressure recovery capacity of diffuser compared to an ideal diffuser of same geometry, which can be evaluated as shown in Equation 3

$$
\xi_{d}=\frac{C_{p s}}{C_{p-i d e a l}}
$$

In order to determine the type of flow in the diffuser, the Reynolds dimensionless number is pertinent and evaluated as shown in Equation 4

$$
\operatorname{Re}=\frac{\rho u_{f g-\text { inlet }} D_{\text {inlet }}}{\mu_{f g-\text { inlet }}}
$$

where, $D_{\text {inlet }}$ is the diffuser flow inlet diameter, and $\mu_{f g \text {-inlet }}$ is the dynamic viscosity of the inlet flue gas considered at inlet temperature. 


\section{Numerical Implementations}

The different 2-D geometries of the diffuser were developed and meshed using ANSYS commercial software. The geometric models were created using the dimension of the diffuser used for preliminary experiment while the modifications are done inside the diffuser channel.

Due to the poor diffusion outlined above, several diffuser models with three guide-vanes were developed and simulated to determine the best guide vane shape and orientation for better thermal and hydraulic diffusion and distribution of the hot flue gas across the width of the diffuser outlet/inlet cross-section of the flue gas flow channel. Three different shapes of guide vanes (a. airfoil shapes, b. block, and c. oval) were considered in the modification of the diffuser design. Geometry design and mesh generation of the three models of the guide vane diffuser of different shapes at $30^{\circ}$ and $45^{\circ}$ guide-vane tilt angle are shown in Figure 4 and Figure 5, respectively.

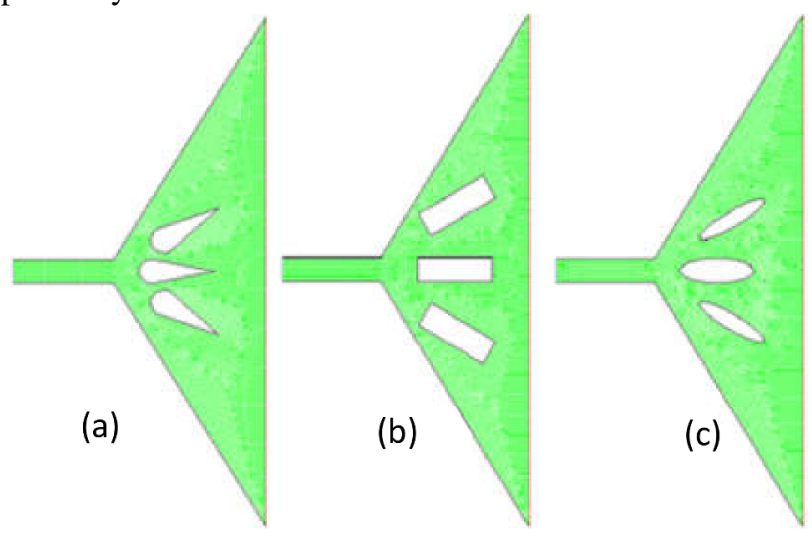

Fig. 4. Different guide-vanes shapes at 30o orientation in the diffuser, (a) Airfoil, (b) Block, and (c) Oval guide vanes.

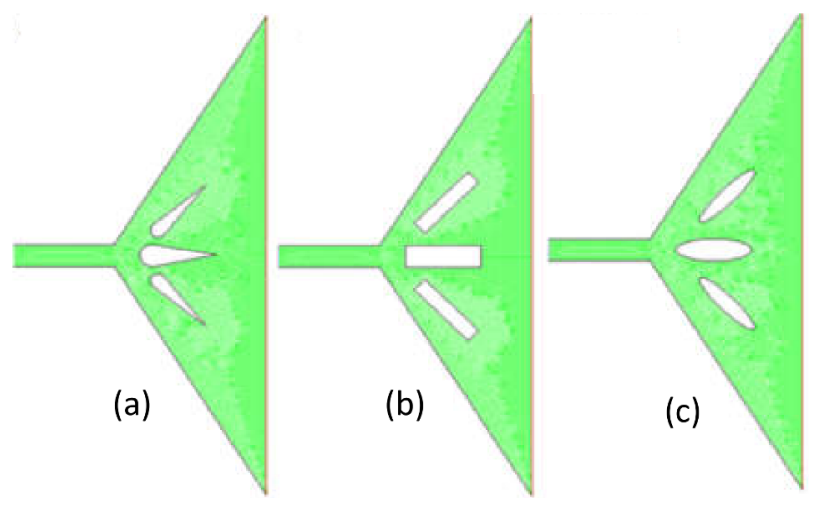

Fig. 5. Different guide-vanes shapes at 450 orientation in the diffuser, (a) Airfoil, (b) Block, and (c) Oval guide vanes.

\subsection{Mesh generation}

ANSYS meshing technology provides a means to balance the time and accuracy requirements and obtain the right mesh for each simulation in the most automated way possible [12]. It also provides the user the option of defining the mesh generation procedure that can help in achieving a better mesh with less skewness. For the 2-D diffuser, the mesh was generated 
such that the mesh density was higher near the walls to resolve the velocity gradients and to meet the requirements of the turbulence model $[12,13]$.

Fig. 6. Five guide vanes model diffuser grid generation.

Thus structured meshing method was employed with size function to generate mesh for the geometry easily. The resulted computational model from the analysis of the performances of the different guide vane shape and orientation angle is shown in Figure 6.

\subsection{Boundary conditions}

Following the geometry dimensions described in Figure 3, the system was modelled for vaneless and guide-vane diffuser. The working fluid in this problem is flue gas produced from experimental gas turbine. The simulation parameters of the diffuser geometry, which are the boundary conditions of the problem, are shown Table 1. All values are assumed constant over the respective component of the boundary. The boundary conditions employed in the simulations was designed to depict what was encountered during the experiment that resulted to the problem.

Table 1. Boundary Conditions adopted for the simulation.

\begin{tabular}{|l|c|l|}
\hline \multicolumn{1}{|c|}{ Component } & Boundary type & \multicolumn{1}{c|}{ Value } \\
\hline Diffuser Wall & Wall & $\mathrm{q}=0 \mathrm{~W} / \mathrm{m}^{2}$, No-slip \\
\hline Guide Vanes & Wall & $\mathrm{q}=0 \mathrm{~W} / \mathrm{m}^{2}$, No-slip \\
\hline \multirow{3}{*}{ Diffuser Inlet } & Velocity inlet & $\mathrm{V}_{\mathrm{fg} \text {-in }}=20 \mathrm{~m} / \mathrm{s}$ \\
\cline { 2 - 3 } & Temperature inlet & $\mathrm{T}_{\mathrm{fg}}$-in $=473 \mathrm{~K}$ \\
\cline { 2 - 3 } & Inlet flow condition & $\mathrm{Re}=30170$ \\
\hline Diffuser Outlet & Pressure outlet & $\mathrm{p}_{\mathrm{o}}=0.0 \mathrm{~Pa}$ \\
\hline
\end{tabular}

At the inlet, a constant velocity distribution in the free stream region is set. The velocity distribution at the wall boundary layers is described by the second order. To isolate the influence of the aspect ratio on the diffuser performance, no boundary layers are applied to the parallel diffuser walls at the inlet. The second order approach is valid for the computations with the standard k- $\varepsilon$ turbulence model. The turbulence parameters at the inlet are set under the assumption of isotropic turbulence, a turbulence intensity of $1.5 \%$ and a hydrodynamic diameter equal the inlet diameter $(0.05 \mathrm{~m})$. At the outlet static pressure condition was used while the normal gradients of the turbulence parameters are set to zero. At the solid walls and the guide vanes, no heat generation and no-slip boundary conditions were applied to all velocity components at the wall. The simulations were performed using the segregated iterative solver of ANSYS-FLUENT. The convergence criteria are set to $10-6$ for the $k$ - $\varepsilon$ computations. 
Due to the unknown variable pressure at the outlet, continuity was employed to formulate an equation by adopting the SIMPLE algorithm. This study used the second order scheme for the spatial and the time derivative discretization. Due to the complexity of surface, a compromise between simplicity, ease of implementation, accuracy and computational efficiency was made and second-order methods were selected with this compromise in mind as suggested by Ferziger and Perić [14].

\subsection{Grid Independency check}

The accuracy of any simulation is determined by the percentage variance of the result with change in the grid size. The density of the grid has significant impact on the accuracy and convergence of computations. The distribution of the grid nodes is non-uniform because of the grid concentration at the regions near the walls of the diffuser models. The different models of the diffuser (vane-less, 3-guide-vane and the 5-guide-vane diffuser models) were meshed and considered for grid independency. Using the airfoil shaped 5-guide-vane diffuser for the grid independence analysis in this study as presented in Table 2, different mesh sizes were considered for flue gas of $473 \mathrm{~K}$ at inlet velocity of $20 \mathrm{~m} / \mathrm{s}$ with Reynolds number of 3 $\mathrm{x} 10^{4}$. The simulated models were classified based on mesh sizes as coarse mesh, medium mesh, fine mesh and finer mesh. The results of the different meshed diffuser were compared with the results from the finer mesh to check variation of the results. The grid dependent parameters which were compared are the diffuser effectiveness $\zeta_{d}$ and average temperature difference between the inlet and outlet.

Table 2. Grid generation and grid independency.

\begin{tabular}{|l|c|c|c|c|}
\hline \multirow{2}{*}{\begin{tabular}{c}
\multirow{2}{*}{$\begin{array}{c}\text { Grid dependent } \\
\text { parameters }\end{array}$} \\
\cline { 2 - 5 }
\end{tabular}} & \multicolumn{4}{|c|}{ Grid Analysis and sizes } \\
\cline { 2 - 5 } & Coarse Mesh & Medium Mesh & Fine Mesh & \multirow{2}{*}{ Finer Mesh } \\
\cline { 2 - 5 } & 1425 & 5689 & 23388 & 133880 \\
\hline Effectiveness $\left(\zeta_{d}\right)$ & 0.0241 & 0.0565 & 0.0704 & 0.0708 \\
\hline Error & 0.0467 & 0.0143 & 0.0004 & - \\
\hline Error $(\%)$ & 66.00 & 20.20 & 0.56 & - \\
\hline Temperature \\
Difference, $\Delta \mathrm{T}$
\end{tabular}

The errors were gradually converging towards zero with respect to mesh size from coarse mesh to fine mesh. The diffuser effectiveness percentage error considered from the finer meshed diffuser geometry was found to be $0.56 \%$ while the percentage error associated to the temperature difference was $3.93 \%$ for the fine mesh. Accordingly the number of meshes (cells) for the domain was considered with mesh size of 23,288 cells. 


\section{Results and discussions}

\subsection{Validation and benchmarking}

Following the problem associated with the vane-less diffuser, a simulation for the vane-less diffuser was performed. The simulation results showed exactly what was encountered through the experiment by displaying a jet-like flow discharge at the diffuser outlet with an aspect ratio 1:3 of inlet to outlet as shown in Figure 7 and Figure 8.
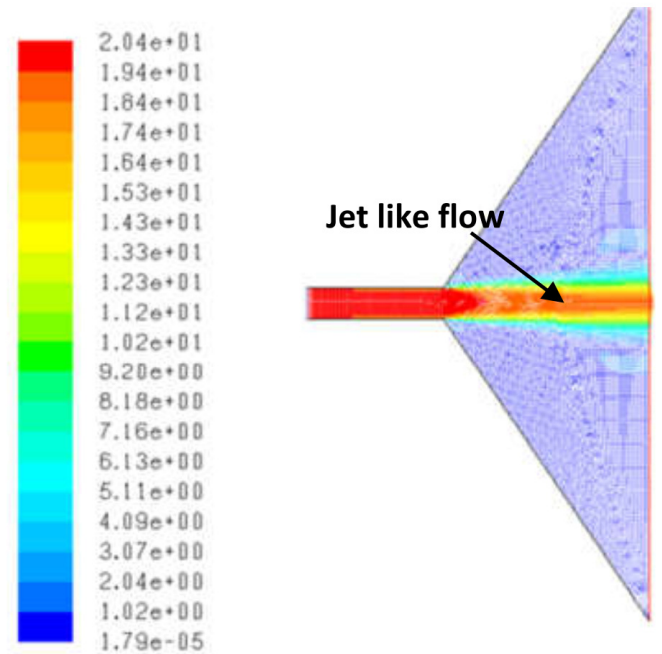

Velocity Vectors Colored By Velocity Magnitude $(\mathrm{m} / \mathrm{s})$

Fig. 7. Velocity vector of jet-like discharge for a vane-less flue gas diffuser.

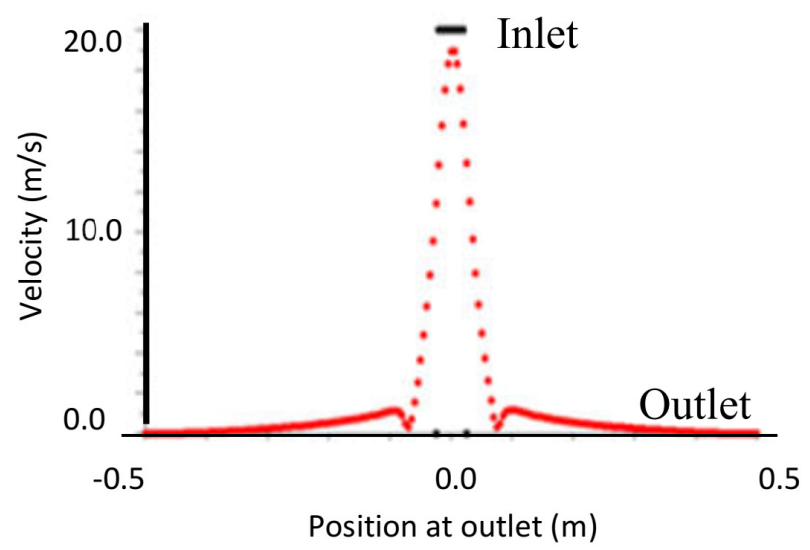

Fig. 8. Flue gas velocity profile at diffuser outlet.

The results also showed that the temperature distribution was concentrated within the jet diffusion area which leads to distortion at the center of the wall of the inclined flue gas channel during the experiment. The temperature contour and distribution at the diffuser outlet is as shown in Figure 9 and Figure 10. 


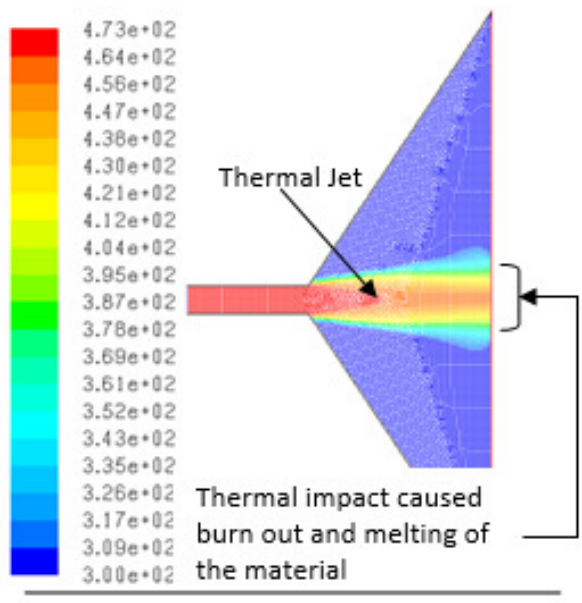

Contours of static temperature (K)

Fig. 9. Flue gas flow temperature of a vane-less flue gas diffuser.

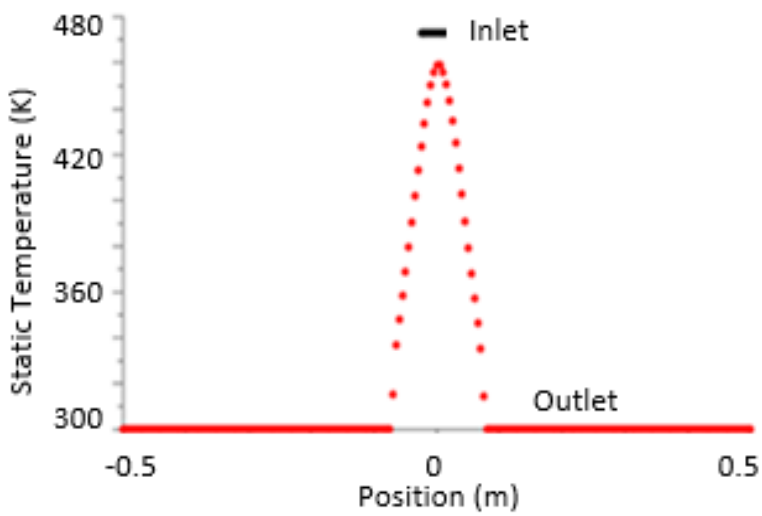

Fig. 10. Flue gas temperature distribution at the inlet and outlet of the vane-less flue gas diffuser.

\subsection{Pressure recovery analysis}

Considering the pressure distribution at the inlet and outlet of the diffuser, Figure 11 shows the distribution and the recoverable pressure using the vane-less diffuser. Even though some pressure could be recovered using this diffuser, there was uneven distribution of the flue-gas across the outlet of the diffuser.

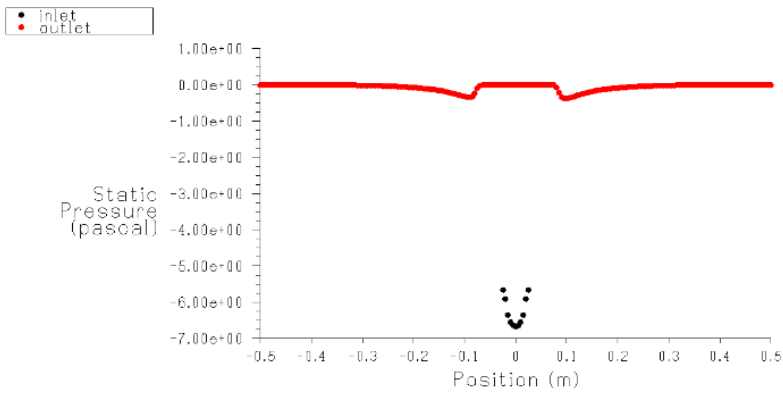

Fig. 11. Flue gas pressure distribution at the inlet and outlet of the vane-less flue gas diffuser. 
The negative pressure at the inlet, as may be noticed in Figure 11, indicates the differences between the operating pressure and the absolute pressure and also as a result of variation in velocity of the flue gas between the inlet and outlet of the diffuser; consequently the static pressure is smaller at inlet than at the outlet.

Considering the vane-less and the $30^{\circ}$ orientated guide-vaned for the different guide-vane shapes, Figure 12 shows the flue gas flow pressure at the diffuser inlet and outlet. The results showed that at the diffuser outlet, the airfoil shaped guide vane offered higher pressure recovery compared to the other guide vane shapes. At the inlet the vane-less diffuser showed lowest pressure compared to other models of $30^{\circ}$ guide-vane orientation. The average pressure difference for the different orientations showed that airfoil guide vanes performed better than block and oval shape guide vane.

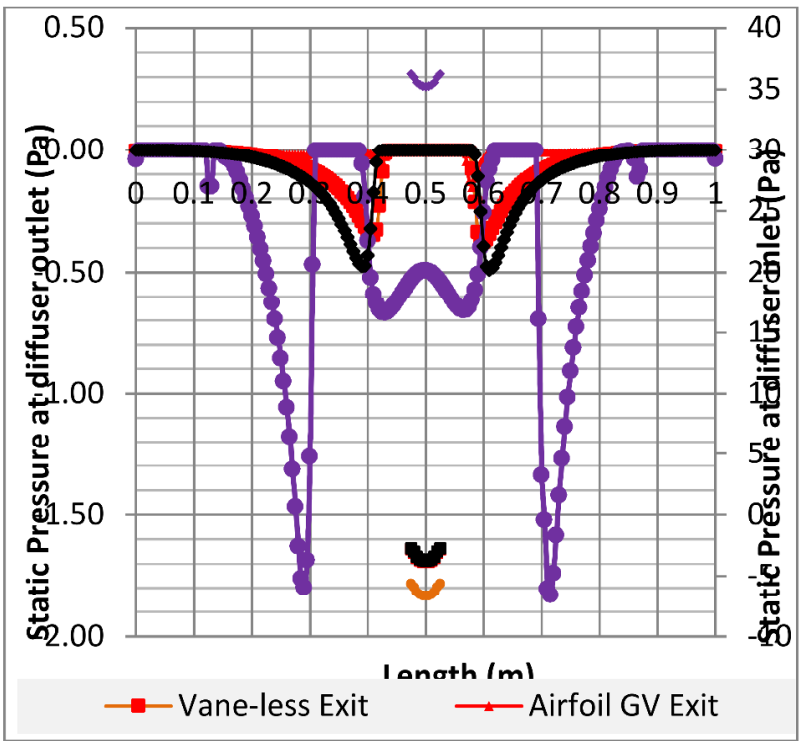

Fig. 12. Diffuser inlet and outlet static pressure for $30^{\circ}$ inclined GV.

Similarly, considering the vane-less and guide-vanned diffuser of $45^{\circ}$ orientations for the various guide-vane shapes. The results based on the average recoverable pressure, Table 3 , at the diffuser outlet using the $45^{\circ}$ inclined guide-vanes showed that the airfoil shaped guidevane offered higher pressure recovery compared to the other guide vane shapes.

Table 3. Pressure at inlet and outlet of vane-less and 450 vane-orientated diffuser models and their pressure difference.

\begin{tabular}{|l|c|c|c|c|}
\cline { 2 - 5 } \multicolumn{1}{c|}{} & \multicolumn{4}{c|}{ Flow guide configuration } \\
\hline Pressure (Pa) at & Vane-less & Airfoil & Block & Oval \\
\hline Inlet Pressure & -6.265 & -10.26 & 1.4126 & -2.9827 \\
\hline Outlet pressure & -0.061 & -0.0597 & -0.0589 & -0.0795 \\
\hline Pressure Difference & 6.204 & 10.2 & 1.472 & 2.903 \\
\hline
\end{tabular}

The pressure recovery is not the only factor required in proper design of this diffuser under consideration with respect to the purpose for which it is planned for, thus temperature distribution and flow velocity are basically very important factors. 


\subsection{Analysis of thermal diffusion}

Considering the flue gas outlet temperature distribution at the diffuser outlet for the $30^{\circ}$ and $45^{\circ}$ guide-vanned diffuser models are presented in Figure 13 and Figure 14 respectively. With the $30^{\circ}$ guide-vane diffuser, the block-GV model showed a better temperature dispersion compared to the airfoil and the oval shaped-GV models.

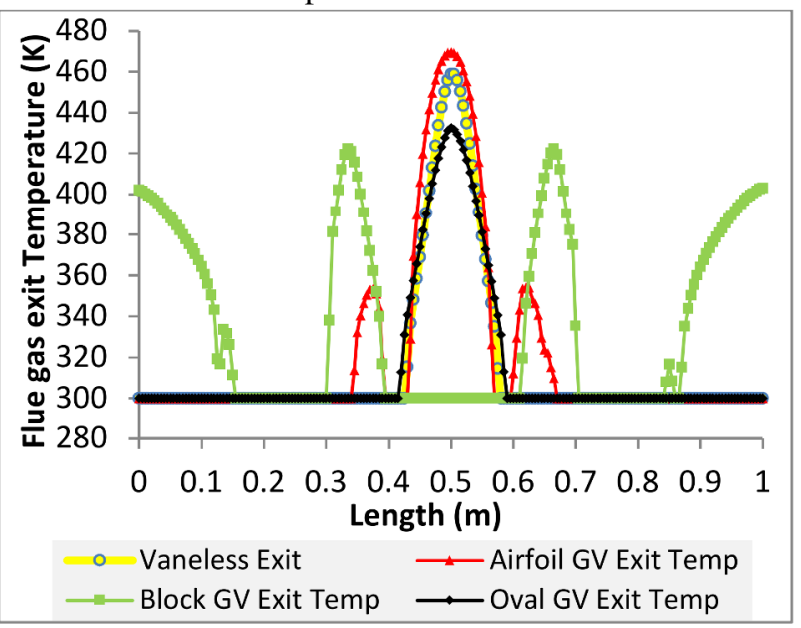

Fig. 13. Flue gas outlet temperature at the diffuser outlet for vane-less and for different shapes of guide vanes inclined at $30^{\circ}$.

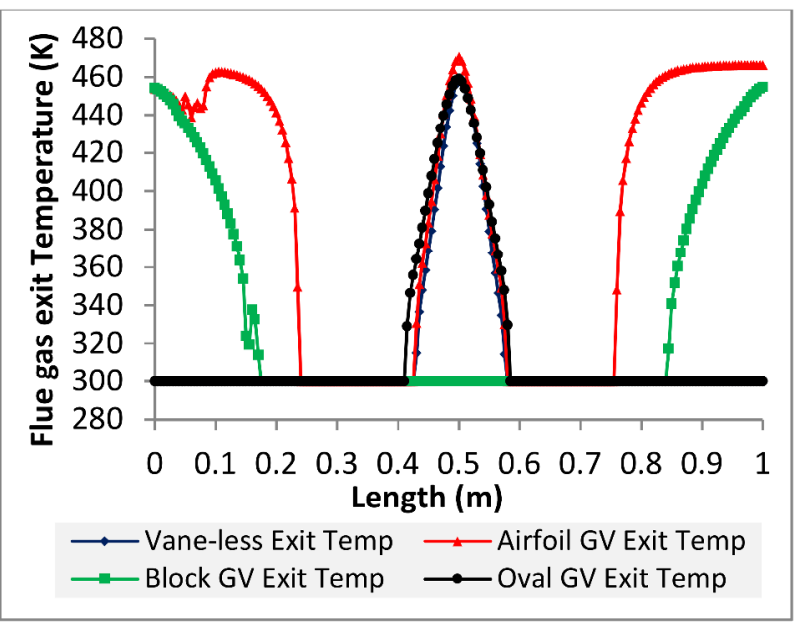

Fig. 14. Flue gas outlet temperature at the diffuser outlet for vane-less and for different shapes of guide vanes inclined at $45^{\circ}$.

The temperature distribution using the $45^{\circ}-\mathrm{GV}$ showed that the airfoil shaped GV model presented a better distribution than block and oval shape GV models.

Considering the flue gas velocity at the diffuser outlet using the various guide-vane shape diffuser models, the velocities of the flue gas at the diffuser outlet of $30^{\circ}$ and $45^{\circ}$ inclinedGV are presented in Figure 15 and Figure 16, respectively. The velocity at the outlet was best using the block-shaped $\mathrm{GV}$ for $30^{\circ} \mathrm{GV}$ models as compared to the oval and airfoil shaped $\mathrm{GV}$ models. On the other hand, the $45^{\circ}$ inclined GV models as shown in Figure 16 shows that the airfoil displays better distribution of flue gas velocity at the diffuser outlet than the block and oval shaped GV models 
Following the variation in the velocity at the flue gas diffuser outlet, the average velocities for the different models computed as shown in Table 4. Even though Figure 16 showed the airfoil to have better distribution for the $45^{\circ}$ guide-vanned diffuser models, the average outlet velocity was lower than the average outlet velocity of the block-GV model.

Table 4. Average FG velocity at the diffuser outlet length for vane-less and guide vane diffuser at $30^{\circ}$ and $45^{\circ}$ orientations.

\begin{tabular}{|c|c|c|c|c|}
\hline \multirow{2}{*}{ GV Orientation } & \multicolumn{4}{|c|}{ Average Air Velocity at Diffuser Outlet (m/s) } \\
\cline { 2 - 5 } & Vane-less & Airfoil GV & Block GV & Oval GV \\
\hline $\mathbf{3 0}^{\mathbf{0}}$ & 1.634 & 1.235 & 3.215 & 1.733 \\
\hline $\mathbf{4 5}^{\mathbf{0}}$ & 1.634 & 1.933 & 2.822 & 1.721 \\
\hline
\end{tabular}

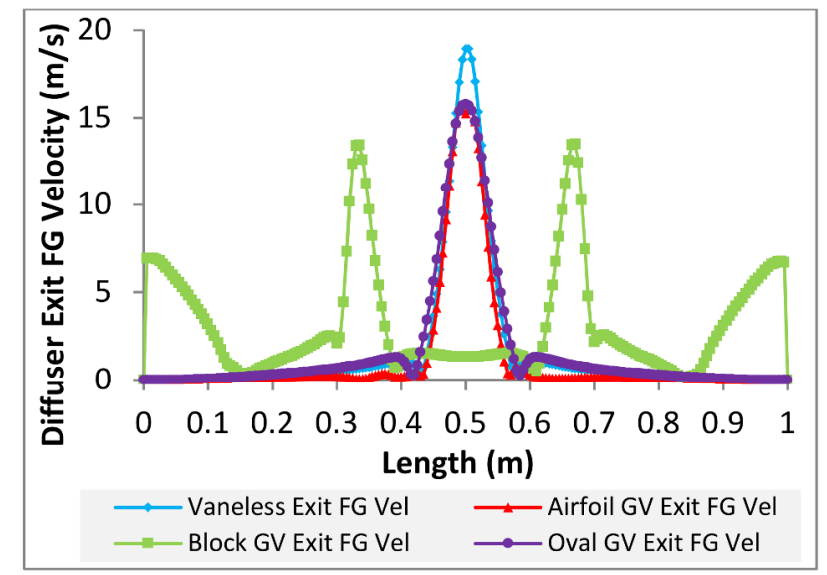

Fig. 15. Flue gas diffuser outlet velocity for vane-less diffuser and diffuser models of different guide vane shapes inclined at $30^{\circ}$.

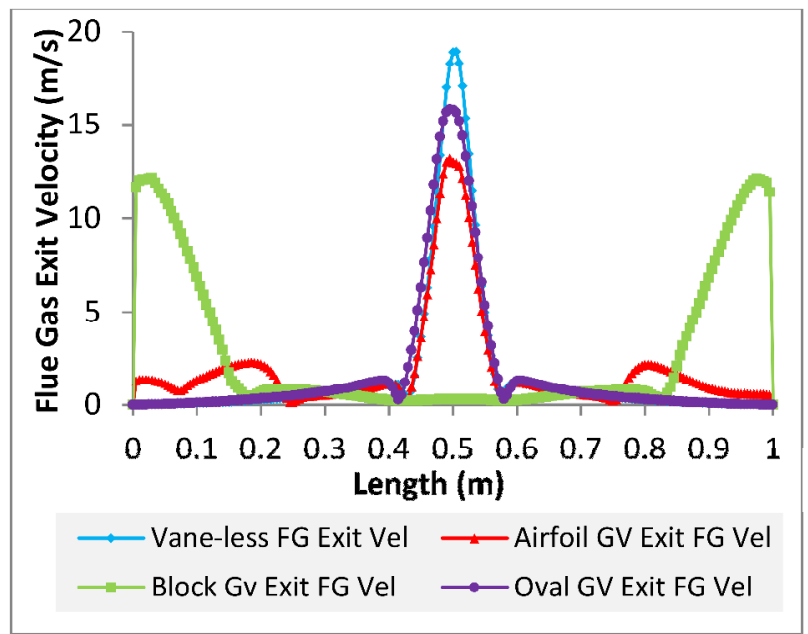

Fig. 16. Flue gas Diffuser outlet velocity for vane-less diffuser and diffuser models of different guide vane shapes inclined at $45^{\circ}$.

The temperature, pressure and velocity of the flue gas at the diffuser outlet are all important in their respective capacity thus percentage weight of importance to the design of this diffuser was assigned for the decision of the best guide vane shape to be used in designing the diffuser with five (5) guide vanes for proper diffusion and distribution of the hot flue gas 
over the cross-sectional area of the diffuser outlet. The weights assigned to temperature, pressure and velocity are $0.4,0.3$ and 0.3 respectively. The reason for the higher weight assigned to temperature is that the diffuser was planned for flue gas distribution across a heat exchanger in the form of flat-plate. Table 5 describes the weight of the different parameters for the different shape of guide vane. The weights were assigned considering the maximum value of the fluid properties at the different guide vane orientation angles. For instance, referring back to Table 4 , the maximum recoverable pressure for guide vanes at $30^{\circ}$ orientation was found to be $3.44 \mathrm{~Pa}$ using airfoil shapes. This was assigned the maximum weight value of 0.4 while for other shape, their values were percentage of the maximum value while the negative values were assigned zero (0) weight.

Table 5. Property weight assignment for GV shape and orientation angle selection.

\begin{tabular}{|l|c|c|c|c|c|c|c|}
\hline \multicolumn{1}{|c|}{ Properties } & CW & \multicolumn{2}{c|}{ Airfoil shape } & \multicolumn{2}{c|}{ Block shape } & \multicolumn{2}{c|}{ Oval shape } \\
\hline & & $30^{\circ}$ & $45^{\circ}$ & $30^{\circ}$ & $45^{\circ}$ & $30^{\circ}$ & $45^{\circ}$ \\
\hline Pressure & 0.3 & 0.3 & 0.3 & 0.0 & 0.00 & 0.28 & 0.1 \\
\hline Temperature & 0.4 & 0.38 & 0.4 & 0.4 & 0.35 & 0.37 & 0.3 \\
\hline Velocity & 0.3 & 0.12 & 0.21 & 0.3 & 0.30 & 0.16 & 0.2 \\
\hline Total & 1. & 0.8 & 0.91 & 0.7 & 0.65 & 0.82 & 0.6 \\
\hline
\end{tabular}

From Table 5, the maximum output based on the assigned weights was found to be the airfoil shape at $45^{\circ}$ inclination while the oval shape and the airfoil at $30^{\circ}$ orientation are almost in same range. For simplicity and consistency, a choice of the airfoil shape for both $30^{\circ}$ and $45^{\circ}$ orientation using five guide vanes was implemented for the diffuser design in this study. Even though the study also simulated combinations of different shapes at different orientation angles but finally it was found that the use of airfoil for the entire guide vanes gave better result The study also further simulated combinations of different shapes at different orientation angles but finally it was found that the use of airfoil for the entire guide vanes gave better result. The orientation angle of the guide vanes was studied for five guide vane configuration and it showed that the best angles are 0o for the center guide vane which has arc radius equal to the diffuser inlet radius and the mid-guide vanes inclined at an angle of $30^{\circ}$ while the extreme guide vanes is best at $45^{\circ}$ orientation from the center-line based on the diffuser under study.

After many trials on the models and designs, it was found that the distance between the center guide vane and the diffuser inlet has a very important role to play in the flow uniformity at the diffuser outlet, in terms of velocity, pressure and temperature distribution. The optimum distance between the inlet of the diffuser to the center guide vane was found to fall within a length less than or equal to the diameter of the diffuser inlet. Similarly it was also found that the radius of the airfoil center guide vane gives best result when its cord radius equals or greater than the radius of the diffuser inlet with maximum radius equals 1.25 times the diffuser inlet radius. In the selection of best configuration, the temperature distribution is the major determinant for this diffuser because the system for which the diffuser is designed is a heat recovery system, thus, a five guide-vane configuration was finally selected as it offers the best result. Figure 17 and Figure 18 show the static temperature and velocity distribution along the diffuser outlet. The $\mathrm{x}$-axis in the figures represent the exit plane, where 0 and 1 are the left and right wall points, and 0.5 is the center of the exit, which is in the same line of the inlet centerline of the gas supply pipe. 


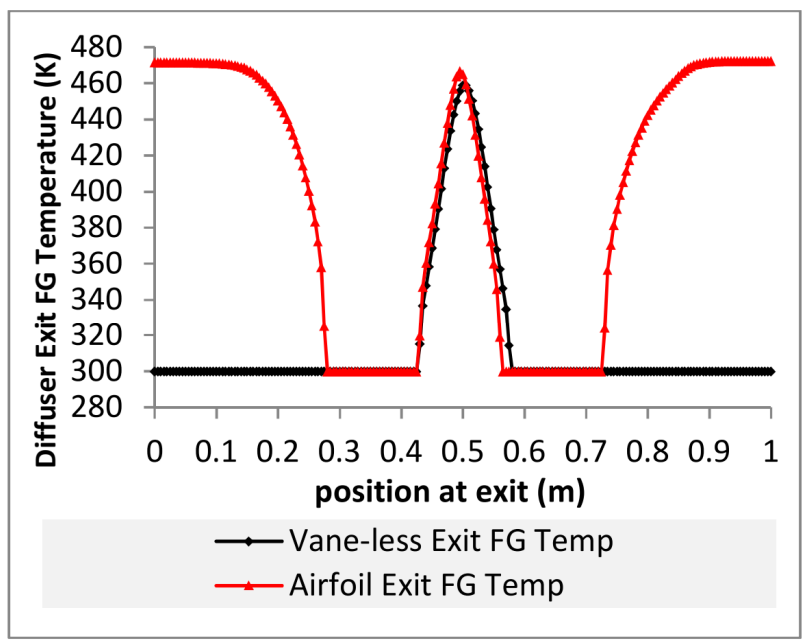

Fig. 17. Static Temperature distribution in the diffuser.

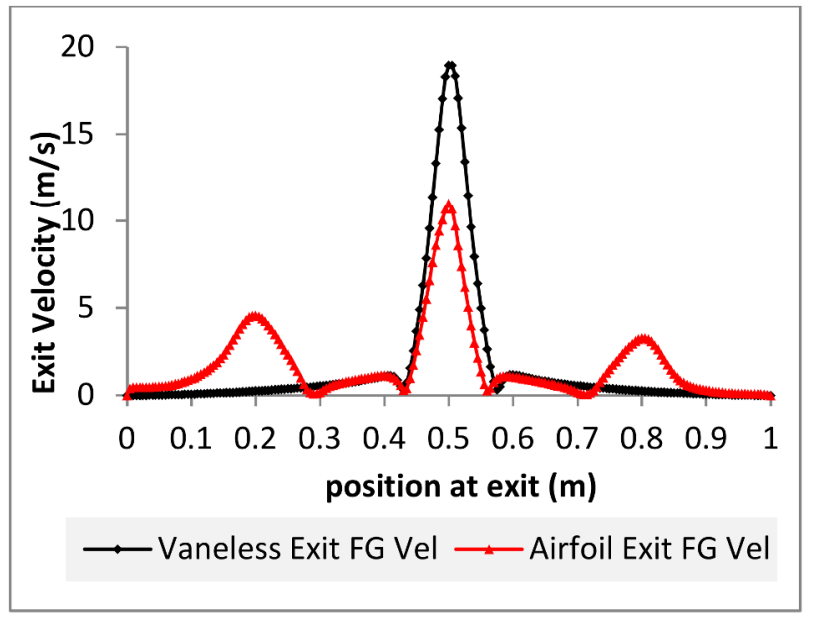

Fig. 18. Static temperature of flue gas at the diffuser outlet of vane-less and five-airfoil shaped guide vane.

The velocity magnitude and static pressure for the 5-GV diffuser model was compared with the vane-less diffuser at the diffuser outlet as shown in Table 6.

Table 6. Parameters.

\begin{tabular}{|c|c|c|c|c|}
\hline \multirow{2}{*}{$\begin{array}{c}\text { GV } \\
\text { Orientation }\end{array}$} & \multicolumn{4}{|c|}{ Average Air Velocity at Diffuser Outlet (m/s) } \\
\cline { 2 - 5 } & Vane-less & Airfoil GV & Block GV & Oval GV \\
\hline $\mathbf{3 0}^{\circ}$ & 1.634 & 1.235 & 3.215 & 1.733 \\
\hline $\mathbf{4 5}^{\mathbf{0}}$ & 1.634 & 1.933 & 2.822 & 1.721 \\
\hline
\end{tabular}

The final achievement from the study, which has been implemented in the system fabrication, is presented in Table 7, where the pressure, temperature and velocity have been improved by $25.5 \%, 26.3 \%$ and $9 \%$, respectively. 
Table 7. Overview of the performance of Vane-less Diffuser and 5-Airfoil GV Diffuser.

\begin{tabular}{|c|c|c|c|}
\hline Parameters & Position & Vane-less & 5 Airfoil GV \\
\hline \multirow{3}{*}{ Average Pressure } & Inlet Static Pressure (Pa) & -6.26 & -7.84 \\
\cline { 2 - 4 } & Outlet Static Pressure (Pa) & -0.06 & -0.05 \\
\cline { 2 - 4 } & Pressure recovered (Pa) & 6.20 & 7.78 \\
\hline \multirow{3}{|c|}{ Improvement (\%) } & $\mathbf{2 5 . 4 3}$ \\
\hline \multirow{2}{*}{$\begin{array}{c}\text { Average } \\
\text { Temperature }\end{array}$} & Inlet Temperature (K) & 473.0 & 473.0 \\
\cline { 2 - 4 } & Outlet Temperature (K) & 314.7 & 397.6 \\
\hline \multicolumn{3}{|c|}{ Improvement (\%) } & 26.34 \\
\hline \multirow{2}{*}{ Average Velocity } & Inlet Velocity (m/s) & 20.0 & 1.78 \\
\cline { 2 - 4 } & Outlet Velocity (m/s) & 1.63 & $\mathbf{9 . 0 5}$ \\
\hline
\end{tabular}

\section{Conclusion}

The 2-D thermal diffuser with aspect ratio of 20 and area ratio of 25 is simulated and investigated in this paper. The diffuser is studied without and with guide vanes. The use of guide vanes for distribution has considerable effect in creating approximately uniform diffusion at the outlet of a diffuser. Different shapes and numbers of guide vanes have been proposed and investigated. Five airfoil shaped distribution guide vanes offer the best diffusion with less pressure loss. The best orientation angles of the distribution guide-vane for a diffuser with diffusion design angle within $45^{\circ}$ to $60^{\circ}$ range are $0^{\circ}, 30^{\circ}$ and $45^{\circ}$ for a five-vane diffuser. With the use of diffusion guide vane, about $85 \%$ uniform diffusion can be achieved.

The authors acknowledge the ministry of higher education, $\mathrm{MOE}$ - Malaysia for providing the financial fund under the project FRGS/2/2010/TK/UTP/03/4. Also acknowledge are goes to Universiti Teknologi PETRONAS, UTP for providing the fund to the research project under STIRF no. 61/09.10. In particular, the second author is highly appreciate UTP for supporting his $\mathrm{PhD}$ study under Graduate Assistance (GA) scheme.

\section{References}

1. L. R. Reneau, J. P. Johnston, and S. J. Kline, J. Fluid Eng., 89, 141-150, (1967).

2. K. Jiao, H. Sun, X. Li, H. Wu, E. Krivitzky, T. Schram, and L. Larosiliere, Proceedings of the Institution of Mechanical Engineers, Part D: Journal of Automobile Engineering, 223, 1061-1070, (2009).

3. M. Marconcini, F. Rubechini, A. Arnone, and S. Ibaraki, J. Turbomach., 132, (2010).

4. M. Kumar Gopaliya, M. Kumar, S. Kumar, and S. Manjaree Gopaliya, Aerosp. Sci. Technol., 11, 130-135, (2007).

5. R. B. Anand and R. Sandeep, Frontiers in Automobile and Mechanical Engineering (FAME2010), 295-300 (2010).

6. M. K. Gopaliya and K. K. Chaudhary, Aerosp. Sci. Technol., 14, 338-347 (2010).

7. N. Arndt, A. Acosta, C. Brennen, and T. Caughey, J. Turbomach., 112, 98-108, (1990).

8. M. A. Aziz, I. A. M. Gad, E. S. F. A. Mohammed, and R. H. Mohammed, Energ. Buildings, (2012).

9. T. Esch and F. R. Menter, Fourth International Symposium on Turbulence, Heat and Mass Transfer, Antalya, Turkey, (2003).

10. F. Menter, M. Kuntz, and R. Langtry, Turbulence, heat and mass transfer, 4, 625-632, (2003). 
11. T. Drabek, Mechanical and Industrial Engineering, Technische Universit Wien, Vienna, Austria, (2001).

12. C. ANSYS, "User Manual, ANSYS" ed: Inc, (2011).

13. I. C. ANSYS, ANSYS 12.0 User Manual. Canonsburg, PA: ANSYS, Canonsburg, PA, (2009).

14. J. H. Ferziger and M. Perić, Computational methods for fluid dynamics vol. 3. Berlin: Springer, (2002). 